\title{
A Case Report of Penetrating Injury of the Abdomen
}

\section{Mojgan Rahimi ${ }^{1 *}$, Pooya Kalani}

${ }^{1}$ Department of Anesthesiology and Critical Care, Tehran University of Medical Sciences, Tehran, Iran

${ }^{2}$ Department of Anesthesiology and Critical Care, Hamedan University of Medical Sciences, Hamedan, Iran

\section{A BSTRACT}

Introduction: Anaesthetic and surgical management of abdominal trauma are often challenging. Case Description: In this case study, we introduced a 10-year-old boy who had a penetrating injury of the abdomen with a metal rod projecting anteriorly. Results: After opening the abdomen, none of the abdominal viscera were injured. Conclusion: Positioning for intubation and cutting the metal rod were discussed.

\section{Key words:}

1. Wounds and Injuries

2. General Surgery

3. Anesthesia

*Corresponding Author: Mojgan Rahimi

E-mail: Drr_anesthesist@yahoo.com 
كزارش يك مورد از تروماى نافذ شكمى

$$
\begin{aligned}
& \text { مزگَان رحيمى "'، يويا كلانى' } \\
& \text { 'كروه بيهوشى و مراقبتهاى ويزّه، دانشعاه علوم يزشكى تهران، تهران، ايران } \\
& \text { "كرّوه بيهوشى و مراقبتهاى ويرّه، دانشكاه علوم يزشكى همدان، همدان، ايران }
\end{aligned}
$$

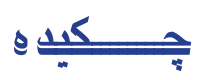

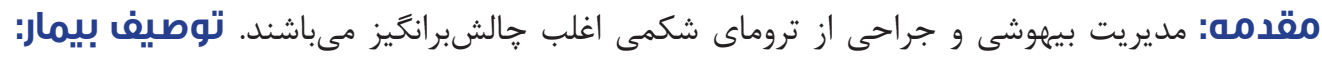

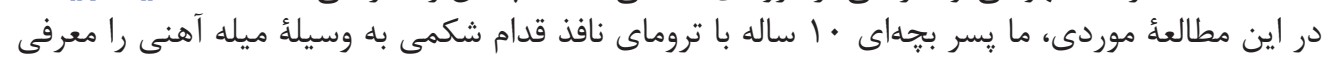

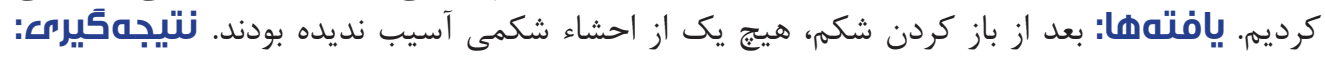

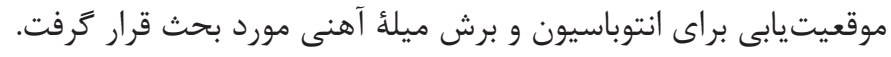

\footnotetext{
كليد وازهها:

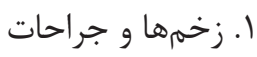

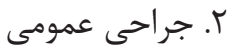

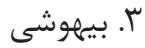

" نويسنده مسئول: مزَّان رحيمى " آدرس الكترونيكى: Drr_anesthesist@yahoo.com 


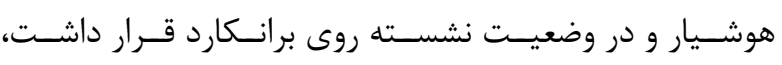

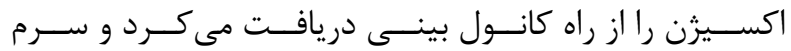

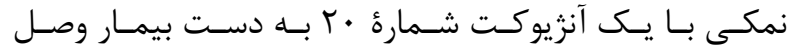

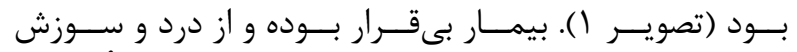

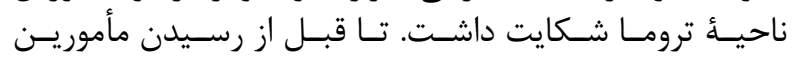

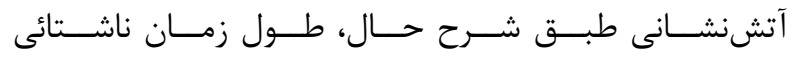

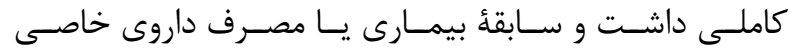

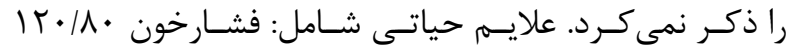

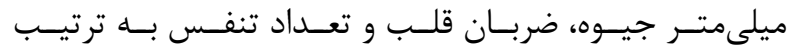

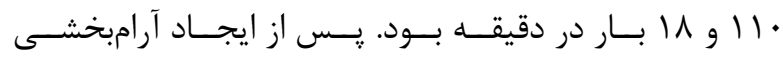

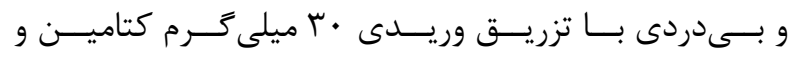

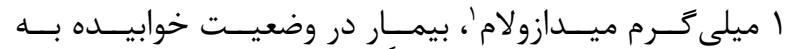

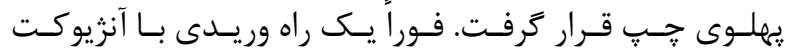

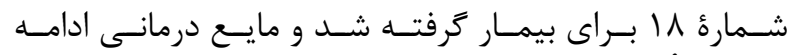

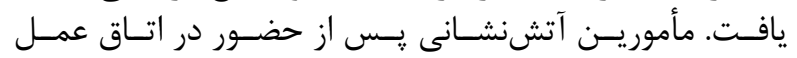

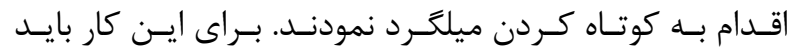

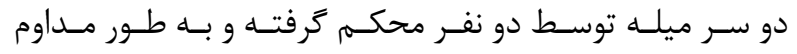

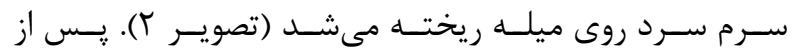

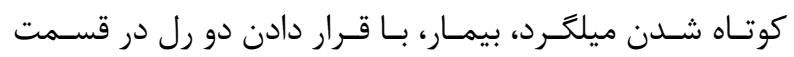

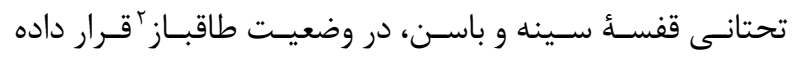

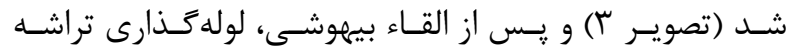

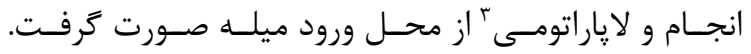
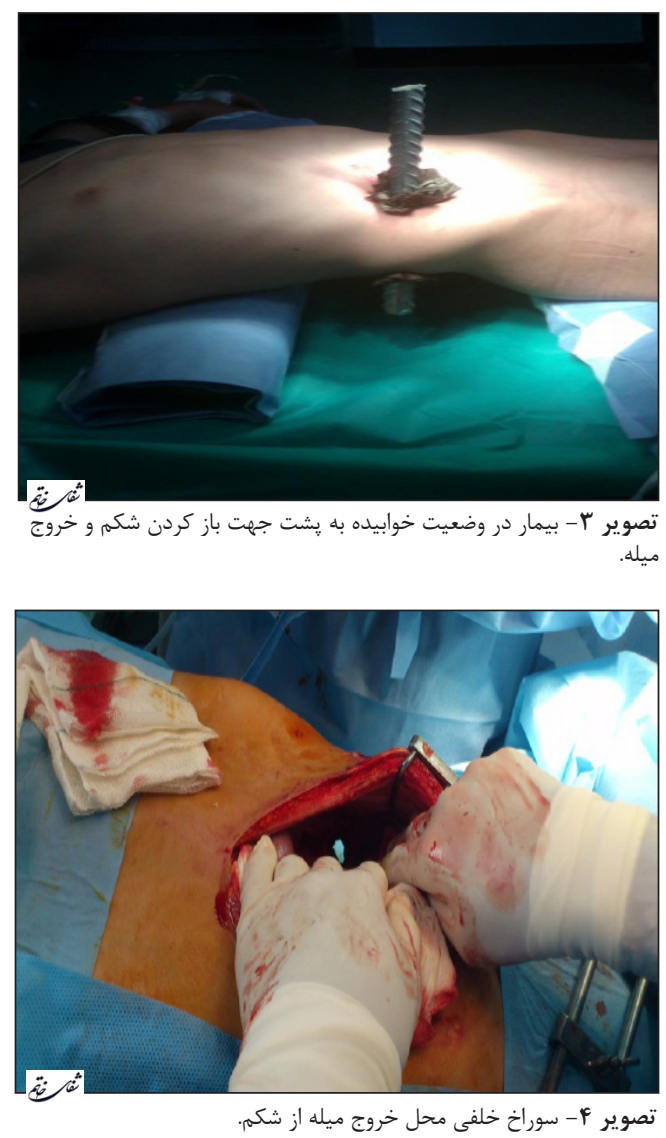

Midazolam

${ }^{2}$ Supine position

${ }^{3}$ Laparotomy

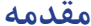

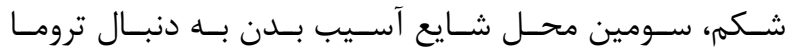

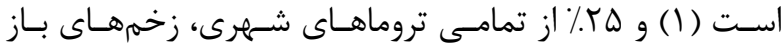

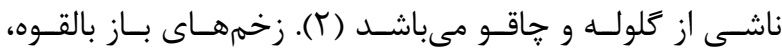

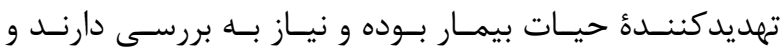

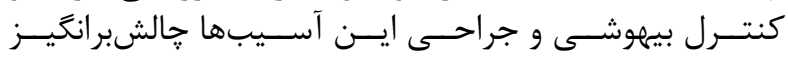

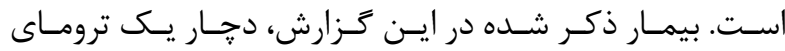

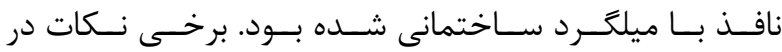

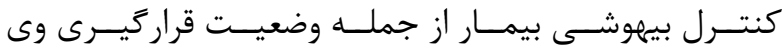

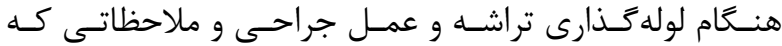

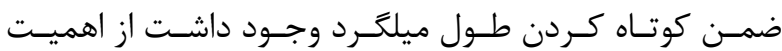

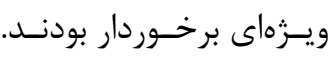
توصيف بيمار

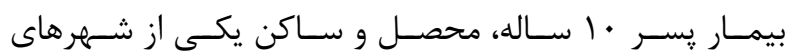

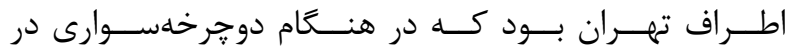

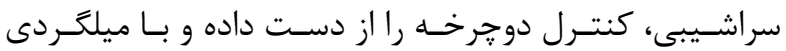

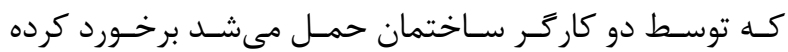

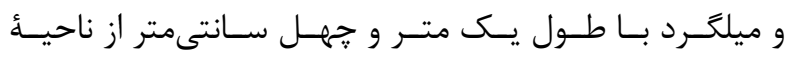

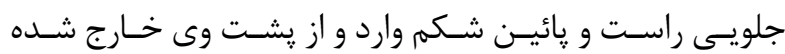

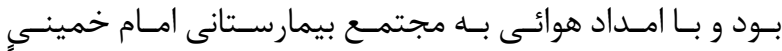

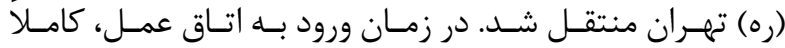
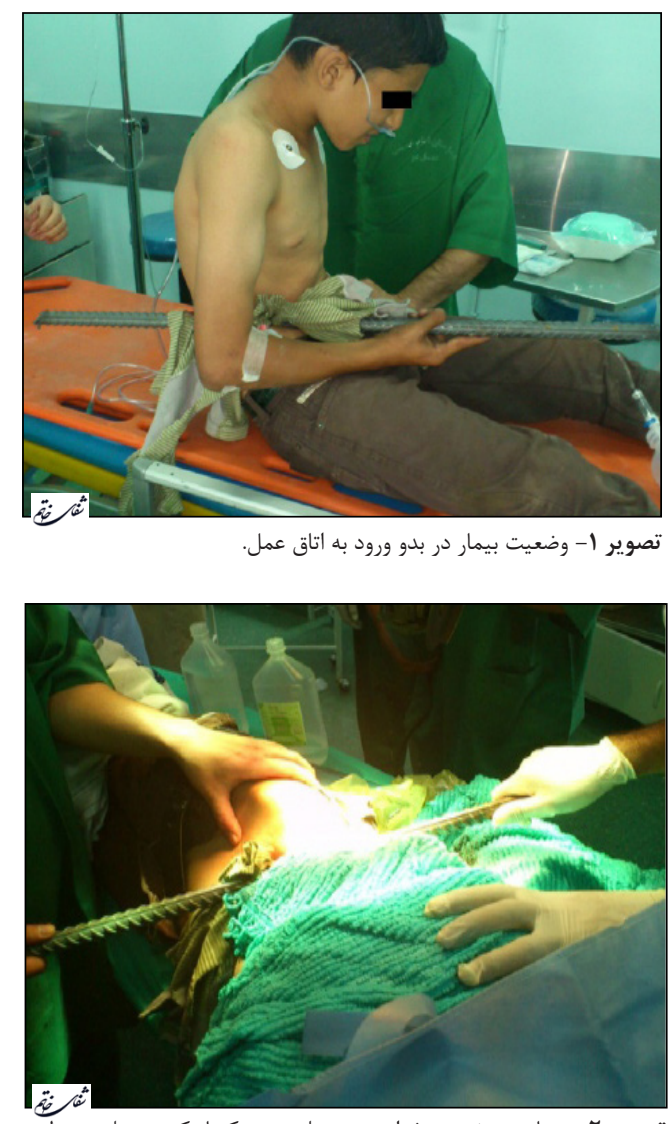

تصوير r- بيمار در وضعيت خوابيده به يهلو جهت كوتاه كردن ميله توسط برسنل آتشنشانى. تصو بيمار 


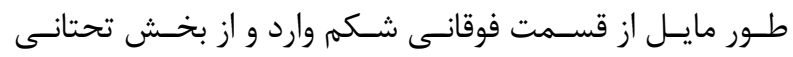

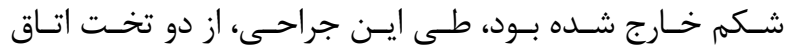

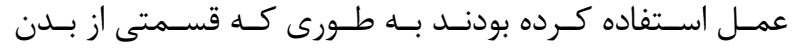

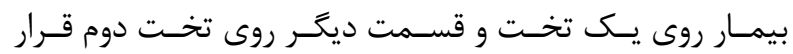

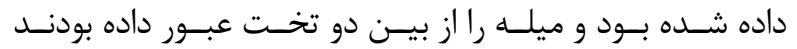

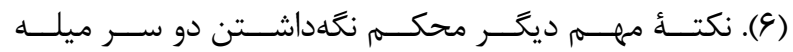

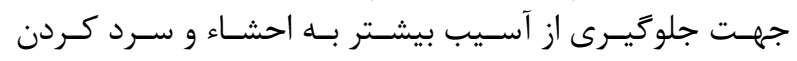

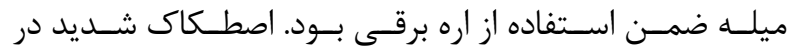

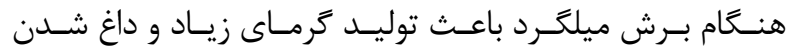

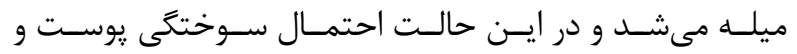

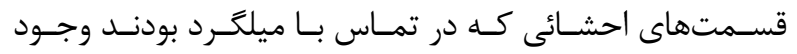

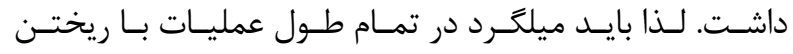

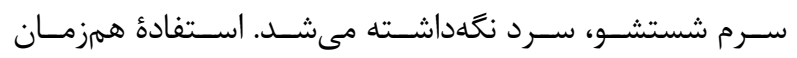

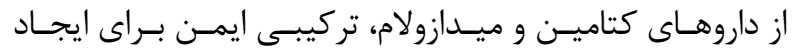

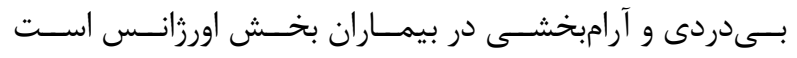

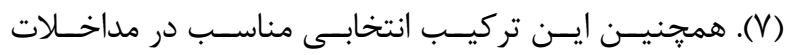

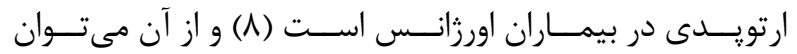

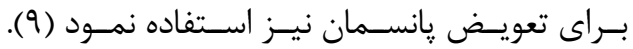
بــه طــور كلـى ملاحظــات خــاص در كنتــرل وضعيـت ايسن

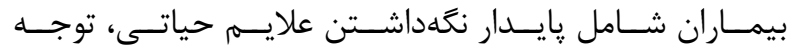

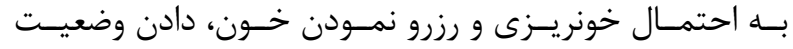

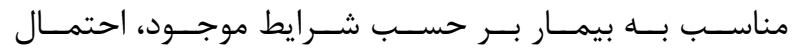

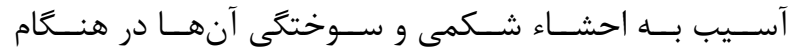

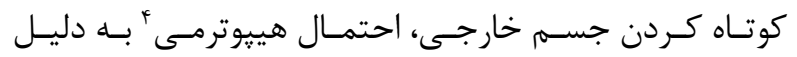

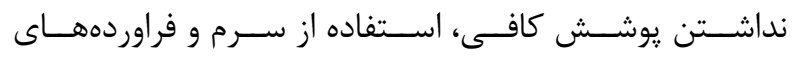

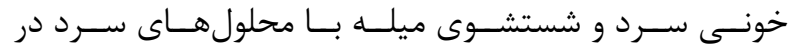

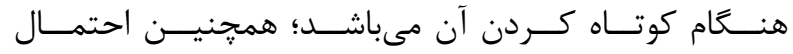

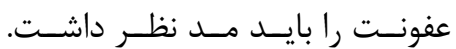

1. Cavina E, Neidhart JP. The trauma of the abdomen: European course of trauma care. [Last accessed on 2010 Aug 12]. Available from: http://www.med.unipi.it/cdu/ ECTC/indexectc.htm.

2. Bowley DM, Robertson SJ, Boffard KD, Bhagawanjee $\mathrm{S}$. Resuscitation and anaesthesia for penetrating trauma. Curr Opin Anaesthesiol. 2003; 16(2): 165-71.

3. Clay Cothren C, Biffle LW, Moore EE. Trauma. Brunicardi F, Andersen D, Billar T, Dunn D, Hunter J, Mathews J, Pollock RE. Trauma Schwartz's Principles of surgery. 9th ed. New York. McGrow-Hill. 2009; p. 135-95.

4. Yong EL, Lim PH, Chng HC, Ng BK. Penetrating wounds of the abdomen and chest: An analysis of 107 consecutive cases. Singapore Med J. 1983; 24(6): 337-42.

5. Panchamia V, Thaker N, Jadeja C, Shah BJ. Emergency anaesthetic management of cardio thoracic and abdominal injury. Indian J Anaesth. 2015; 59(3): 186-7.
بافتها

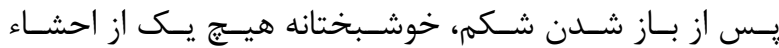

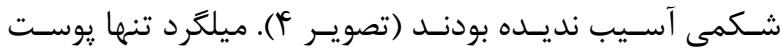

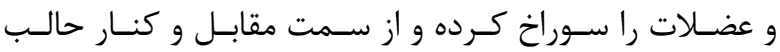

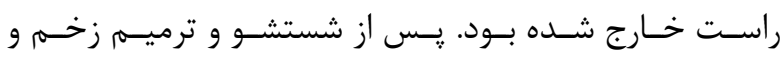

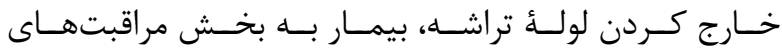

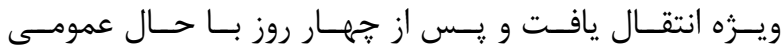

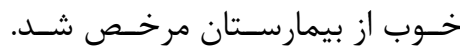
بحث و نتيجه كيرى

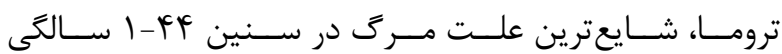

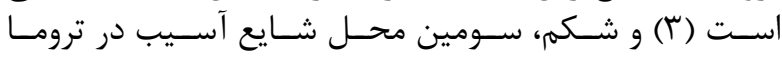

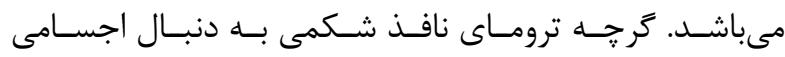

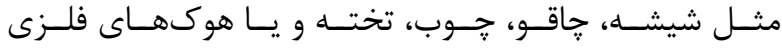

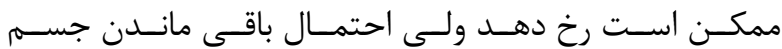

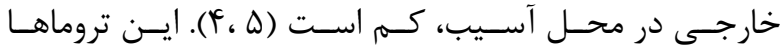

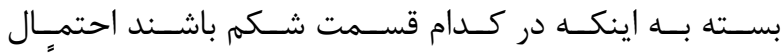

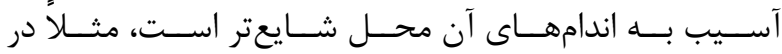

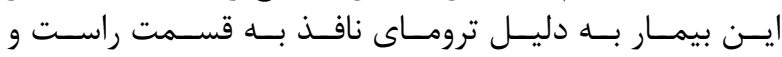

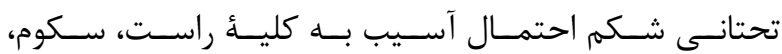

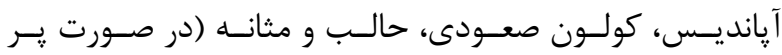

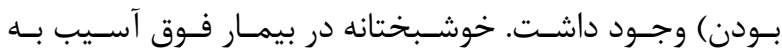

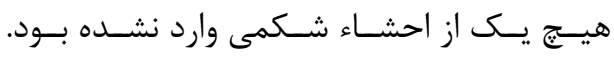

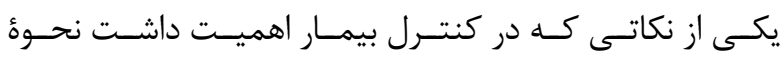

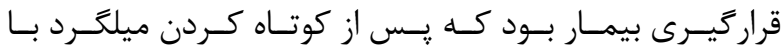

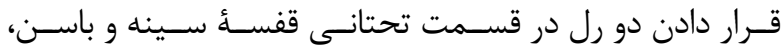

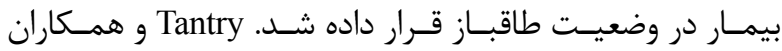

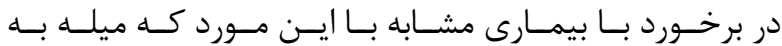

منابع

6. Tantry TP, Kadam D, Shetty 1P, Adappa KK, Muralishankar BG and Shenoy SP. Penetrating abdominal injury in a polytrauma patient: Anaesthetic challenges faced. J Anaesthesiol Clin Pharmacol. 2011; 27(2): 272-4.

7. Chudnofsky CR, Weber JE, Stoyanoff PJ, Colone PD, Wilkerson MD, Hallinen, et al. A combination of midazolam and ketamine for procedural sedation and analgesia in adult emergency department patients. Acad Emerg Med. 2000; 7(3): 228-35.

8. Kennedy RM, Porter FL, Miller JP, Jaffe DM. Comparison of fentanyl/midazolam with ketamine/ midazolam for pediatric orthopedic emergencies. Pediatrics. 1998; 102(4 Pt 1): 956-63.

9. Sih K, Campbell SG, Tallon JM, Magee K, Zed PJ. Ketamine in adult emergency medicine: controversies and recent advances. Ann Pharmacother. 2011; 45(12): 1525-34. 